\title{
Increased Sphingosine Kinase 1 Expression Is Associated with Poor Prognosis in Human Solid Tumors: A Meta-Analysis
}

\author{
Chuanmeng Zhang $\mathbb{D}^{1},{ }^{1}$ Chenglin Zhou $\mathbb{D}^{2},^{2}$ Jie Xu $\mathbb{D}^{1},{ }^{1}$ and Shanshan Xue $\mathbb{D}^{2}$ \\ ${ }^{1}$ The Center for Translational Medicine, Taizhou People's Hospital, Affiliated 5 to Nantong University, Taizhou, \\ 225300 Jiangsu Province, China \\ ${ }^{2}$ Department of Clinical Laboratory, Taizhou People's Hospital, Affiliated 5 to Nantong University, Taizhou, \\ 225300 Jiangsu Province, China
}

Correspondence should be addressed to Shanshan Xue; 245116999@qq.com

Received 20 August 2021; Accepted 12 January 2022; Published 28 January 2022

Academic Editor: Alexander G Mathioudakis

Copyright (c) 2022 Chuanmeng Zhang et al. This is an open access article distributed under the Creative Commons Attribution License, which permits unrestricted use, distribution, and reproduction in any medium, provided the original work is properly cited.

Background and Aim. Sphingosine kinase 1 (SPHK1) is a key enzyme of sphingolipid metabolism which is involved in the pathogenesis and progression of human cancer. It has been demonstrated to be upregulated in various types of human malignancies. However, the prognostic value of SPHK1 remains unclear. Therefore, we performed this meta-analysis to assess its predictive value in the prognosis of cancer patients. Methods. PubMed, Web of Science, Embase, CNKI, and Wanfang databases were thoroughly searched for eligible studies, in which the relationship between SPHK1 expression and cancer prognosis was evaluated. Hazard ratios (HRs) and 95\% confidence intervals (CIs) were pooled to estimate the impact of SPHK1 expression on cancer patients' survival. Odds ratios (ORs) and 95\% CIs were combined to assess the association between SPHK1 expression and clinicopathological characteristics of cancer patients. The certainty of evidence was evaluated by Grading of Recommendations, Assessment, Development, and Evaluation (GRADE) criteria. Results. Thirty studies comprising 32 cohorts with 5965 patients were included in this meta-analysis. The outcomes indicated that elevated SPHK1 expression was associated with worse overall survival (OS) $(\mathrm{HR}=1.71,95 \%$ CI: 1.45-2.01, $P<0.001)$ and disease-free survival (DFS) (HR=1.34, 95\% CI: 1.13-1.59, $P=0.001)$. What is more, SPHK1 overexpression was significantly correlated with certain phenotypes of tumor aggressiveness, such as clinical stage $(\mathrm{OR}=2.07,95 \% \mathrm{CI}: 1.39-3.09, P<0.001)$, tumor invasion $(\mathrm{OR}=2.16,95 \% \mathrm{CI}: 1.47-3.18, P<0.001)$, lymph node metastasis $(\mathrm{OR}=2.04,95 \% \mathrm{CI}: 1.71-2.44, P<0.001)$, and distant metastasis $(\mathrm{OR}=3.16,95 \% \mathrm{CI}: 2.44-4.09, P<0.001)$. The quality of the evidence for both OS and DFS was low. Conclusions. Increased SPHK1 expression is related to poor prognosis in human cancers and may serve as a promising prognostic marker and therapeutic target for malignant patients. However, conclusions need to be treated with caution because of lack of high quality of evidence.

\section{Introduction}

Cancer is a serious public health problem, leading to a severe burden of disease in the world. According to a report released by GLOBOCAN in 2018, approximately 18.1 million people were newly diagnosed with cancer and 9.6 million people died of cancer, which was based on researches performed in 185 countries [1]. The mortality rate for all cancers combined continuously decreased 26\% from 1991 to 2015, and the main reasons included improvements in cancer prevention, screen- ing and early detection, and cancer treatment [2]. However, the 5-year overall survival rate is still low in the majority of cancer patients $[3,4]$. Therefore, it is of great significance to find new biomarkers for the diagnosis and prognosis of cancers.

Sphingolipids are a key component of cancer development due to their role in angiogenesis and lymphangiogenesis driven by cancer stem cells [5]. Many researches have investigated the role of different sphingolipid enzymes, sphingolipid binding proteins, and transmembrane transporters in human 
cancer [6]. Among them, members of the sphingosine kinase (SPHK) family are key enzymes in cancer biology, because their catalytic activity is essential for regulating sphingolipid metabolism [5]. SPHK1, an isoenzyme of SPHK, catalyzes the phosphorylation of sphingosine to form sphingosine-1phosphate (S1P), which inhibits cell apoptosis and promotes cell proliferation and angiogenesis $[7,8]$. In contrast, sphingosine and ceramide, the metabolic precursors of S1P, stimulate apoptosis and inhibit cell proliferation [9]. Thus, the balance between these precursors and S1P within the cell has been proposed as a switch that drives the decision between cell proliferation and death [10]. In addition, the key regulator of this switch is SPHK1, which converts sphingosine into prosurvival S1P $[11,12]$.

SPHK1 has been shown to be significantly upregulated in a variety of cancers, such as breast cancer [5], lung cancer [13], head and neck carcinoma [14], and gastric cancer [15], which may be used as a procancer factor and therapeutic target and have an impact on diagnosis and treatment. Moreover, recent studies have demonstrated that high SPHK1 protein expression is associated with poor prognosis of many tumors [5, 15-35]. However, several publications showed that the association is nonsignificant $[5,16,36-43]$. Therefore, we conducted this meta-analysis to better assess the prognostic value of SPHK1 in tumors.

\section{Materials and Methods}

2.1. Literature Search. In accordance with the Preferred Reporting Items for Systematic Reviews and Meta-Analyses (PRISMA) statement, we performed a systematic literature search of PubMed, Web of Science, Embase, CNKI, and Wanfang databases to find relevant articles assessing the relationship between SPHK1 expression and prognosis of various malignant tumors. The following terms and their combinations are used as search keywords: "sphingosine kinase 1" or "SPHK1") AND ("immunohistochemistry" or "IHC") AND ("cancer" or "tumor" or "neoplasms" or "carcinoma" or "malignancy") AND ("prognosis" or "survival" or "outcome"), covering all articles published in English and Chinese as of July 2021. In addition, we manually screened the reference lists of the retrieved articles to seek for additional eligible studies.

2.2. Inclusion and Exclusion Criteria. Eligible articles in this meta-analysis were subject to the following inclusion criteria: (1) the publication investigated the relationship between SPHK1 expression and the prognosis (overall survival (OS) and disease-free survival (DFS)) of patients with solid tumors. (2) The expression of SPHK1 was detected in the primary cancer tissue by immunohistochemistry (IHC) stain and divided into "positive" and "negative" or "high" and "low" groups; and (3) hazard ratio (HR) and 95\% confidence interval (CI) were readily available or could be calculated indirectly. Studies with any of the following flaws were excluded: (1) reviews, abstracts, letters, editorials, expert opinions, case reports, or animal experiments; (2) HR and 95\% CI could not be obtained by sufficient information or data; and (3) studies with a sample size of less than 50.
2.3. Data Extraction and Quality Assessment. The data extraction in this meta-analysis was independently completed by two investigators (XSS and ZCM) independently using a standardized data-extract form and any divergence was adjudicated through discussion. The following data were collected: first author's name, publication year and language, study region, duration time, cancer type, sample size, followup time, detection method, cut-off value, number and proportion of patients with high SPHK1 expression, survival data, analysis method, and clinicopathological characteristics. If both univariate and multivariate analyses were used to calculate the HR for OS or DFS, the latter was preferred because the result was adjusted for confounding factors and was more accurate [44]. For the articles in which prognosis was plotted only as the Kaplan-Meier curves, the Engauge Digitizer V4.1 software was then applied to obtain survival data according to the method of Tierney et al. [45].

The quality of each included cohort study was independently assessed using the Newcastle-Quality Assessment Scale (NOS). According to the NOS scale, the quality of the cohort study is judged based on three contents: selection of the exposed and unexposed cohort (4 points), comparability of the two cohorts ( 2 points), and outcome assessment (3 points) [46]. Studies with a score $\geq 6$ were considered as of high quality.

2.4. Quality of Evidence. The Grading of Recommendations Assessment, Development, and Evaluation (GRADE) method was applied to evaluate the quality of the evidence $[47,48]$. The GRADE was based on the study design, risk of bias, inconsistency, indirectness, imprecision, and other considerations (including large effect, plausible confounders, and dose-response gradient) [49]. The quality of evidence was graded as high, moderate, low, or very low. The GRADE was assessed using the website http://gradepro.org.

2.5. Statistical Analysis. HRs and their 95\% CIs were combined to estimate the effect of SPHK1 expression on survival. ORs and their 95\% CIs were pooled to assess the association between SPHK1 expression and clinicopathological features. The heterogeneity was evaluated using Cochran's $Q$ and $I$ -squared statistical tests, in which $P<0.05$ or $I^{2} \geq 50 \%$ was considered significant heterogeneity [50]. When significant heterogeneity existed, the random effects model was used for analysis, including subgroup analysis. Otherwise, the fixed effects model was applied. Subgroup analysis was performed to further evaluate the prognostic value of SPHK1. Sensitivity analysis was performed by omitting each cohort study in turn to assess the impact of single cohort on the combined results. Potential publication bias was quantitatively evaluated using Begg's and Egger's asymmetry tests and visually evaluated by funnel plots [51]. If publication bias was found, the trim and fill method was performed to validate the reliability of the meta-analysis results. All analyses were conducted using STATA version 12.0 software (Stata Corporation, College Station, TX), and $P<0.05$ was considered statistically significant. 


\section{Results}

3.1. Literature Search and Study Demographics. The literature search flow diagram is presented in Figure 1. A total of 257 publications were initially retrieved from the PubMed, Web of Science, EMBASE, CNKI, and WanFang database. After removing duplicates and obviously irrelevant research, 73 articles were further screened. Then, 29 papers were excluded by screening the titles and abstracts. Of the remaining 44 potentially relevant articles, 14 studies were excluded because they did not fulfill the inclusion criteria or met one of the exclusion criteria. Finally, 30 studies with 32 cohorts were included in the meta-analysis.

The main characteristics of the 32 eligible cohorts are summarized in Table 1 . Among the 32 cohorts, 5965 patients were included, with samples sizes that ranged from 51 to 1005 . The publication years of the included cohorts ranged from 2008 to 2021. The large majority of cohorts were performed in Asia (twenty in China [15, 17-25, 27-33, 35, 41], two in Korea [5, 37], one in Japan [38], and one in Taiwan [26]), followed by Europe (two in Czech Republic [16], two in UK $[34,43]$, and one in Germany [42]), America (one in Canada [36] and one in USA [39]), and finally Australasia (one in Australia [40]). Among these included cohorts, 17 different cancer types were evaluated, including 5 breast cancer (BC) $[24,34$, 40, 42, 43], 4 gastric cancer (GC) [15, 18, 32], 4 lung cancer (LC) $[16,29,30], 3$ colorectal cancer (CRC) [19, 20, 37, 39], 3 hepatocellular carcinoma (HCC) $[22,23,28]$, and 1 each of ovarian carcinoma (OC) [36], papillary thyroid carcinoma (PTC) [17], renal cell carcinoma (RCC) [21], oral squamous cell carcinoma (OSCC) [38], pancreatic cancer (PC) [25], nasopharyngeal carcinoma (NPC) [27], cervical cancer (CC) [5], cholangiocarcinoma (CCA) [26], bladder cancer (BLC) [41], esophageal carcinoma (ESCC) [31], salivary gland carcinoma (SGC) [33], and astrocytomas (AC) [35]. The expression of SPHK1 was detected by IHC. 29 cohorts reported the correlation between SPHK1 expression and OS $[5,15,16$, 18-33, 35-42], while 11 cohorts evaluated the relationship between SPHK1 expression and DFS [5, 16, 17, 23, 24, 28, $34,40,42,43]$. According to the NOS score, each cohort included in this study gained a score of 6 or more, indicating that the articles were of high quality.

3.2. Association between SPHK1 Expression and Prognosis. As shown in Table 2, a comprehensive analysis was conducted to assess the prognostic value of SPHK1 in human cancer. Twenty-nine cohorts comprising 5466 patients reported the association between SPHK1 expression and OS. The combined HRs indicated that high SPHK1 expression was obviously associated with poor OS ( $\mathrm{HR}=1.71,95 \% \mathrm{CI}$ : $1.45-$ 2.01, $P<0.001)$ using the random-effects model because of heterogeneity $\left(I^{2}=84.3 \%, P<0.001\right)$ (Figure 2$)$. To further examine the prognostic value of SPHK1, subgroup analyses using random-effects model were performed by cancer type, sample size, proportion of patients with high SPHK1 expression, and analysis method. Subgroup analysis of cancer type showed that increased SPHK1 expression was significantly related to poor OS in patients with digestive system malignancies $(\mathrm{HR}=1.79,95 \% \mathrm{CI}: 1.39-2.31, P<0.001)$, urinary system

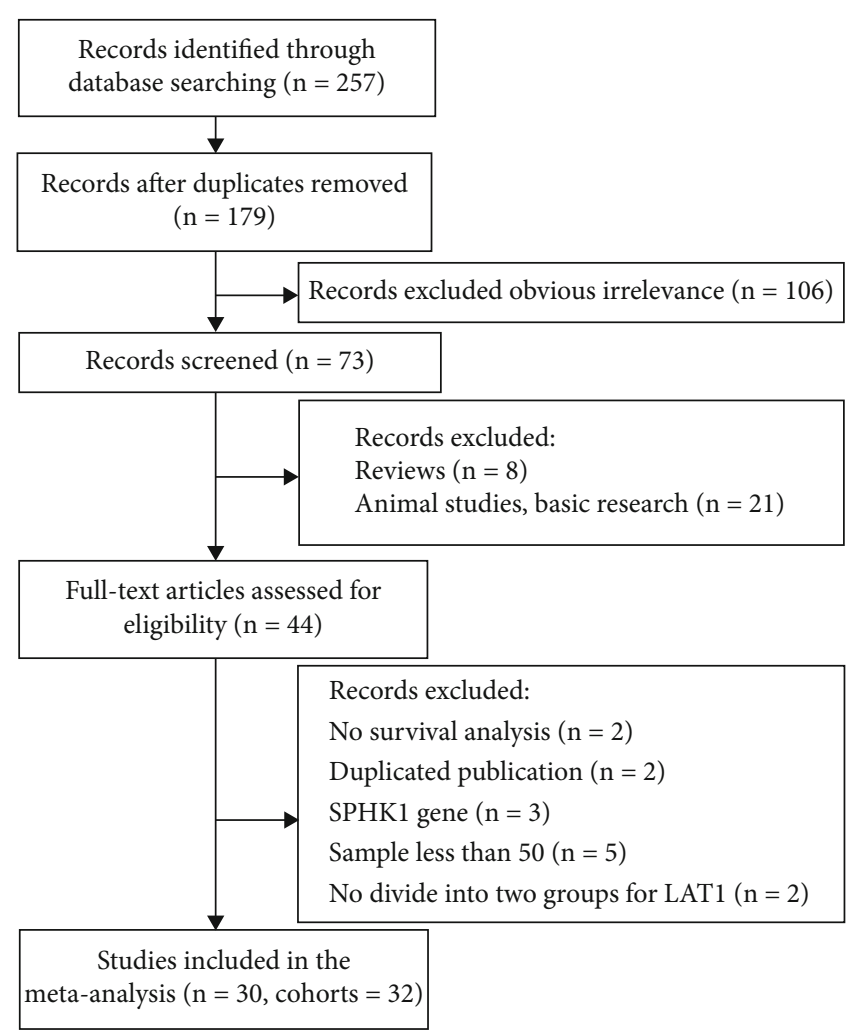

FIgURE 1: Flow diagram of the study selection process and specific reasons for exclusion in the meta-analysis.

cancers $(\mathrm{HR}=1.49,95 \% \mathrm{CI}: 1.20-1.84, P<0.001)$, head and neck cancers $(\mathrm{HR}=2.08,95 \% \mathrm{CI}: 1.48-2.91, P<0.001)$, and LC $(\mathrm{HR}=2.15,95 \%$ CI $1.39-3.35, P=0.001)$, but no significant relationship was observed in patients with reproductive system tumors $(\mathrm{HR}=1.71,95 \% \mathrm{CI}: 0.34-8.64, P=0.519)$ and $\mathrm{BC}(\mathrm{HR}=1.16,95 \% \mathrm{CI}: 0.66-2.02, P=0.608)$. In term of sample size, SPHK1 positive expression was significantly associated with poor OS in the subgroups with large ( $\mathrm{HR}=1.45,95 \% \quad \mathrm{CI}: 1.17-1.80, \quad P=0.001)$ and small (HR $=1.97,95 \% \mathrm{CI}: 1.58-2.45, P<0.001)$ sample sizes. With regard to the proportion of patients with high SPHK1 expression, high SPHK1 expression predicted shorter OS in both high $(\mathrm{HR}=2.07,95 \% \mathrm{CI}: 1.55-2.75, P<0.001)$ and low $(\mathrm{HR}=1.44,95 \% \mathrm{CI}: 1.18-1.76, P<0.001)$ proportion subgroups. Similarly, SPHK1 overexpression was associated with poor OS in the subgroup of analysis method. Thus, almost all subgroup analyses showed that SPHK1 positive expression was associated with poor OS, which to some extent indicates the prognostic value of SPHK1 for tumors and the stability of the results of this study.

Eleven cohorts including 1839 cancer patients reported the impact of SPHK1 on DFS. Due to the obvious statistical heterogeneity $\left(I^{2}=57.3 \%, P=0.009\right)$, the random model was applied and a significant association was observed between increased SPHK1 expression and poor DFS of cancer patients $(\mathrm{HR}=1.34,95 \% \mathrm{CI}: 1.13-1.59, \quad P=0.001)$ (Figure 3). 


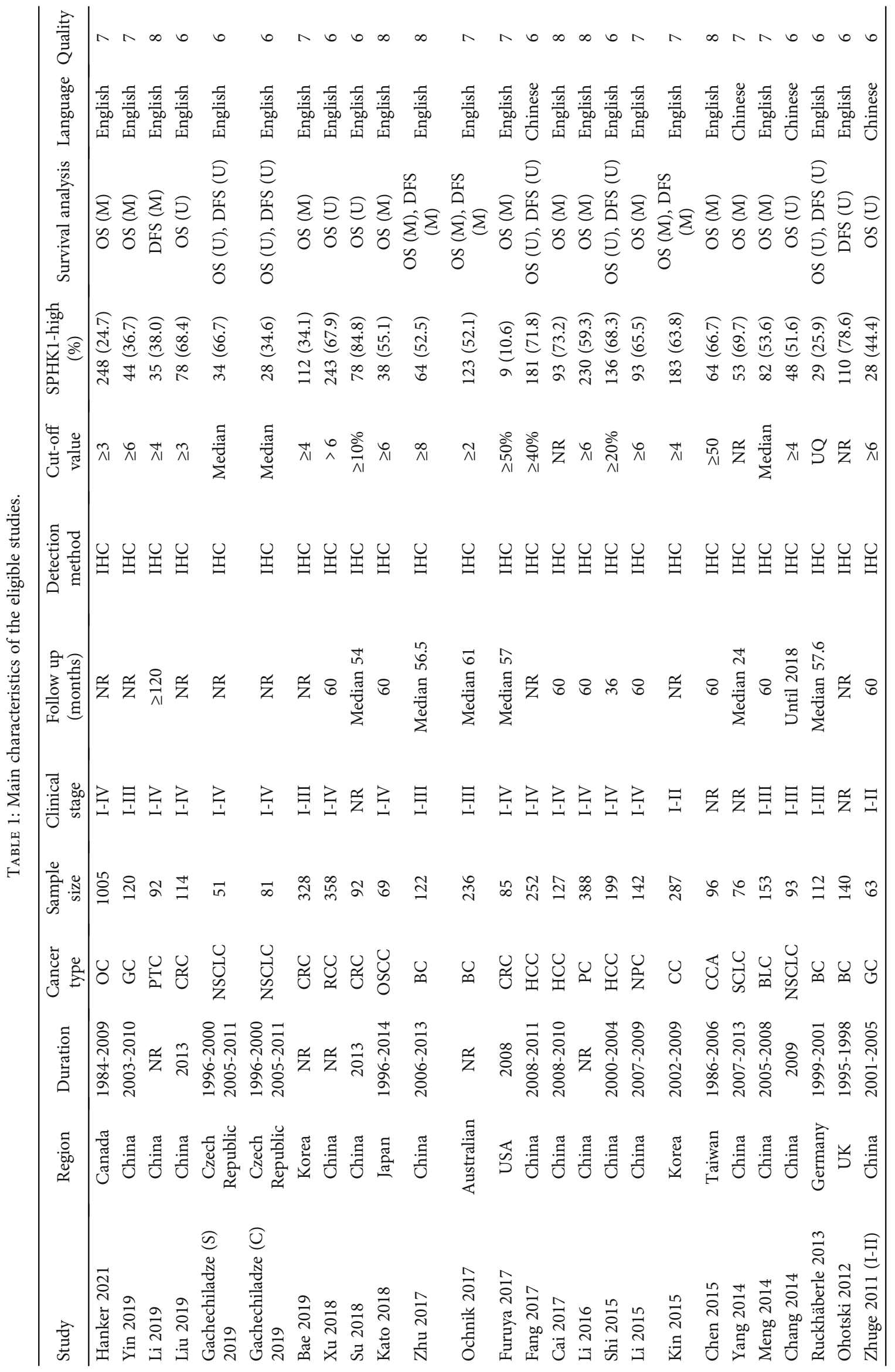




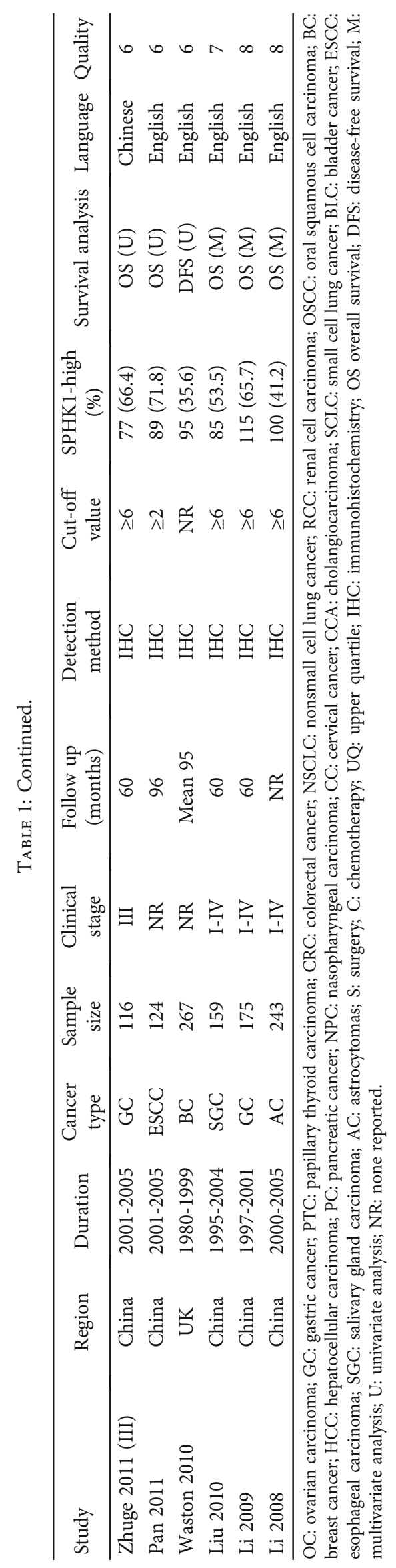


TABLE 2: Summary of the meta-analysis results.

\begin{tabular}{|c|c|c|c|c|c|c|}
\hline Categories & Cohorts (patients) & HR (95\% CI) & $I^{2}(\%)$ & $P_{h}$ & $Z$ & $P$ \\
\hline OS (all) & $29(5466)$ & $1.71(1.45-2.01)$ & 84.3 & $<0.001$ & 6.45 & $<0.001$ \\
\hline \multicolumn{7}{|l|}{ Cancer type } \\
\hline Digestive system & $14(2279)$ & $1.79(1.39-2.31)$ & 89.0 & $<0.001$ & 4.50 & $<0.001$ \\
\hline Urinary system & $2(511)$ & $1.49(1.20-1.84)$ & 0.0 & 0.998 & 3.68 & $<0.001$ \\
\hline Reproductive system & $2(1292)$ & $1.71(0.34-8.64)$ & 64.2 & 0.095 & 0.64 & 0.519 \\
\hline $\mathrm{HNC}$ & $4(613)$ & $2.08(1.48-2.91)$ & 0.0 & 0.841 & 4.26 & $<0.001$ \\
\hline $\mathrm{LC}$ & $4(301)$ & $2.15(1.39-3.35)$ & 63.4 & 0.042 & 3.41 & 0.001 \\
\hline $\mathrm{BC}$ & $3(470)$ & $1.16(0.66-2.02)$ & 74.0 & 0.021 & 0.51 & 0.608 \\
\hline \multicolumn{7}{|l|}{ Sample size } \\
\hline$\geq 150$ & $12(3783)$ & $1.45(1.17-1.80)$ & 88.6 & $<0.001$ & 3.42 & 0.001 \\
\hline$<150$ & $17(1683)$ & $1.97(1.58-2.45)$ & 59.5 & 0.001 & 6.07 & $<0.001$ \\
\hline \multicolumn{7}{|l|}{ SPHK1-high } \\
\hline$\geq 60 \%$ & $14(2209)$ & $2.07(1.55-2.75)$ & 90.4 & $<0.001$ & 4.99 & $<0.001$ \\
\hline$<60 \%$ & $15(3257)$ & $1.44(1.18-1.76)$ & 66.7 & $<0.001$ & 3.64 & $<0.001$ \\
\hline \multicolumn{7}{|l|}{ Analysis method } \\
\hline Multivariate & $17(3811)$ & $1.65(1.34-2.04)$ & 79.1 & $<0.001$ & 4.73 & $<0.001$ \\
\hline Univariate & $12(1655)$ & $1.73(1.43-2.08)$ & 66.3 & 0.001 & 5.75 & $<0.001$ \\
\hline DFS (all) & $11(1839)$ & $1.34(1.13-1.59)$ & 57.3 & 0.009 & 3.36 & 0.001 \\
\hline
\end{tabular}

All pooled HRs were calculated from random-effect model. HNC: head and neck cancer; LC: lung cancer; BC: breast cancer; OS overall survival; DFS: diseasefree survival; HR: hazard ratio; CI: confidence interval; $P: P$ value for statistical significance based on $Z$ test; $P_{h}: P$ value for heterogeneity based on $Q$ test.

3.3. Association between SPHK1 Expression and Clinicopathological Features. The relationship between SPHK1 expression and clinicopathological features is shown in Table 3. Seventeen cohorts with 3951 patients reported the association between SPHK1 expression and clinical stage, and the combined result indicated that high SPHK1 expression was obviously related to clinical stage (I-II vs. III-IV) $(\mathrm{OR}=2.07,95 \% \mathrm{CI}: 1.39-3.09, P<0.001)$. Similar results showed that high SPHK1 expression was significantly associated with tumor invasion (T1-T2 vs. T3-T4) $(\mathrm{OR}=2.16$, 95\% CI: 1.47-3.18, $P<0.001$ ), lymph node metastasis (negative vs. positive) $(\mathrm{OR}=2.04,95 \% \mathrm{CI}: 1.71-2.44, P<0.001)$, and distant metastasis (negative vs. positive) $(\mathrm{OR}=3.16$, 95\% CI: 2.44-4.09, $P<0.001)$. This obvious association was not observed in age (young vs. old) $(\mathrm{OR}=1.47,95 \% \mathrm{CI}$ : $0.80-2.70, P=0.217)$, gender (male vs. female) $(\mathrm{OR}=1.02$, $95 \%$ CI: $0.87-1.19, P=0.813$ ), and tumor size (small vs. large) $(\mathrm{OR}=1.36,95 \%$ CI: 0.85-2.18, $P=0.199)$.

3.4. Sensitivity Analysis and Publication Bias. Sensitivity analysis was conducted to evaluate the influence of each cohort on the meta-analysis results of OS by omitting one cohort in turn. The results confirmed the robustness and reliability about the prognostic value of high SPHK1 expression on unfavorable OS (Figure 4(a)). Similarly, there was no significant change after omitting any cohort on the impact of SPHK1 expression on DFS, indicating the stability of our meta-analysis (Figure 4(b)).

Both Begg's and Egger's tests were used to assess the potential publication bias of OS and DFS. For OS, the funnel plot showed a certain degree of asymmetry and was also confirmed by the Egger's test $(P<0.001)$, while the $P$ value of the Bgger's test was greater than $0.05(P=0.399)$. Thus, the trim and fill method was used. Nine missing cohorts were needed to fill into the funnel plot (Figure 5(b)), and the adjusted $\mathrm{HR}(\mathrm{HR}=1.42,95 \% \mathrm{CI}=1.21-1.65, P<0.001)$ still showed the significant relationship between SPHK1 overexpression and worse OS, indicating the reliability of our results. In addition, there was no potential publication bias for DFS (Begg's test, $P=0.062$; Egger' test, $P=0.082$ ) (Figure 5(c)).

3.5. Level of Evidence. The GRADE method was adopted to assess the certainty of the evidence. The results indicated that the quality of the evidence for both OS and DFS was low (Table 4). Thus, our confidence in the effect estimate is limited: the true effect may be substantially different from the estimate of the effect.

\section{Discussion}

To date, some original articles have studied the prognostic significance of SPHK1 in solid tumors, and both significant and insignificant studies have emphasized the importance of SPHK1 for survival, so it is necessary to quantitatively summarize the survival results. The current meta-analysis included 32 cohorts with 5965 patients, and the systematically evaluated outcomes indicated that high SPHK1 expression was significantly associated with poor $\mathrm{OS}(\mathrm{HR}=1.71$, 95\% CI: $1.45-2.01, P<0.001)$ and DFS (HR $=1.34,95 \% \mathrm{CI}$ : $1.13-1.59, P=0.001)$ in various tumors. Moreover, sensitivity analysis and publication reinforced the stableness and reliability of the meta-analysis results. However, the quality of the evidence for both OS and DFS was low due to observational studies and heterogeneity. In addition, in order to 


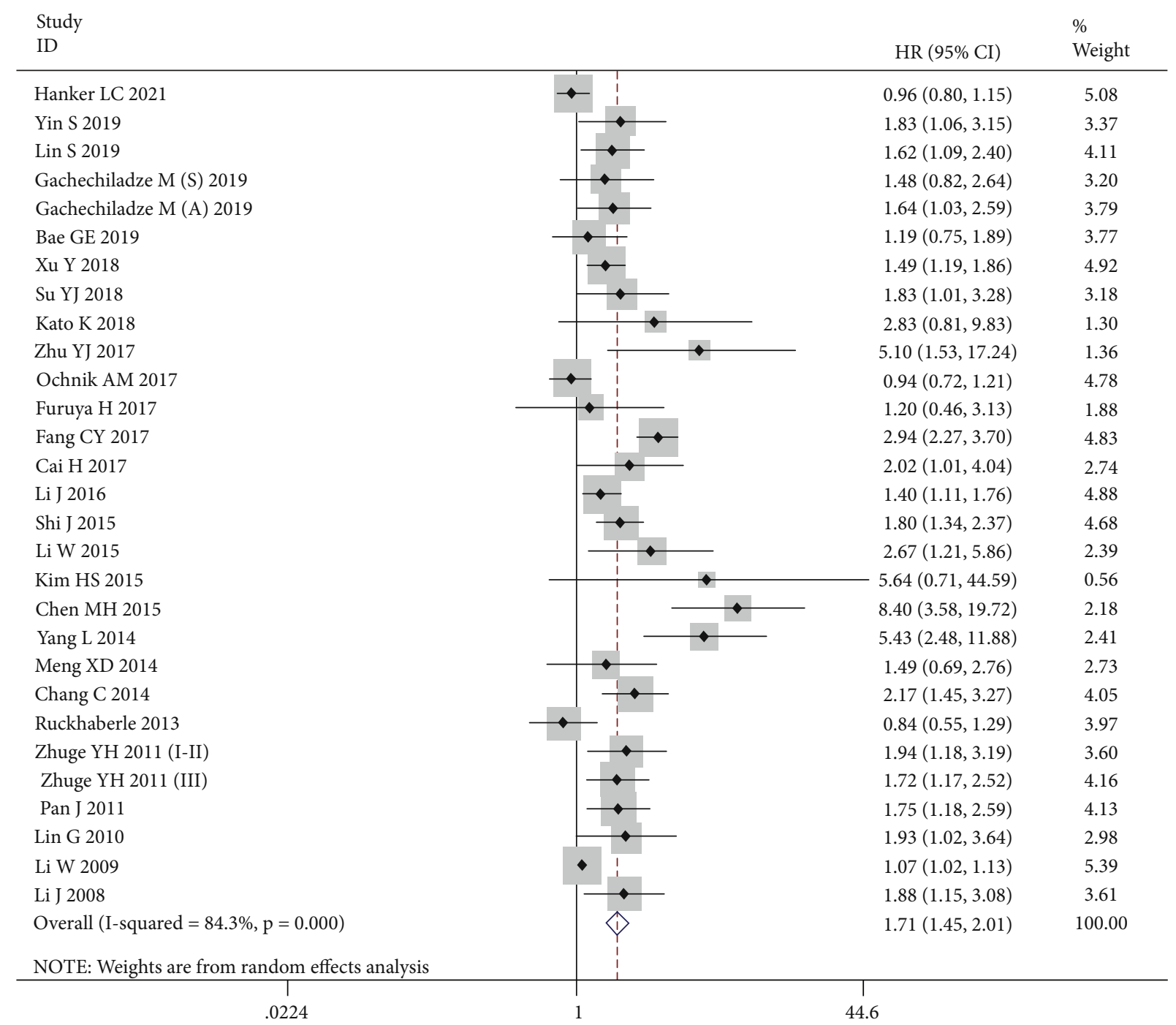

Figure 2: Forest plots of the overall outcomes for overall survival.

further study the prognostic value of SPHK1 in solid tumors, we analyzed the relationship between SPHK1 and clinicopathological features that are also related to the prognosis of cancer patients. The pooled results demonstrated that SPHK1 overexpression was significantly related to clinical stage, tumor invasion, lymph node metastasis, and distant metastasis. Therefore, increased SPHK1 expression may be statistically associated with poor prognosis in cancer patients, although the certainty of evidence is low.

Studies have shown that the abnormal expression of SPHK1 can promote the occurrence of cancer and tumor progression. For example, SPHK1 regulates cell proliferation and apoptosis. SPHK1 can be activated by various growth factors, cytokines, and mitogens, such as vascular endothelial growth factor, platelet-derived growth factor, and tumor necrosis factor- $\alpha[52,53]$. The phosphorylation of SPHK1 and its subsequent translocation from the cytoplasm to the cell membrane were reported to be key factors in the acquisition of malignant phenotypes of cells through promoting the proliferation of malignant cells and protecting the apoptotic pathway from being destroyed [54]. Overexpression of SPHK1 promoted the proliferation in several tumor types, while blockade of SPHK1 inhibited tumor growth [55-58]. Moreover, changing the subcellular localization of SPHK1 had a significant impact on cell function, with cell membrane-translocated exhibiting an effective inhibitory effect on the G1-S phase transition of 3T3-L1 fibroblasts, indicating that the localization of SPHK1 in cells may play a crucial role in the apoptosis of tumor cells [17].

In addition, SPHK1 is also one of the important molecules involved in the invasion and metastasis of malignant tumors. Epithelial-mesenchymal transition (EMT) is originally a developmental procedure that has been used by tumor cells to promote their migration, invasion, and eventually colonization at a distance, resulting in distant metastasis, which leads to a poor prognosis of cancer patients [59]. Loss of E-cadherin and acquisition of vimentin are two critical steps in EMT [60]. Previous studies confirmed that overexpression of SPHK1 was associated with decreased expression of E-cadherin and increased expression of vimentin, suggesting that SPHK1 plays a pivotal role in the EMT of invasive carcinoma cells $[19,38,61]$. In addition, it was 


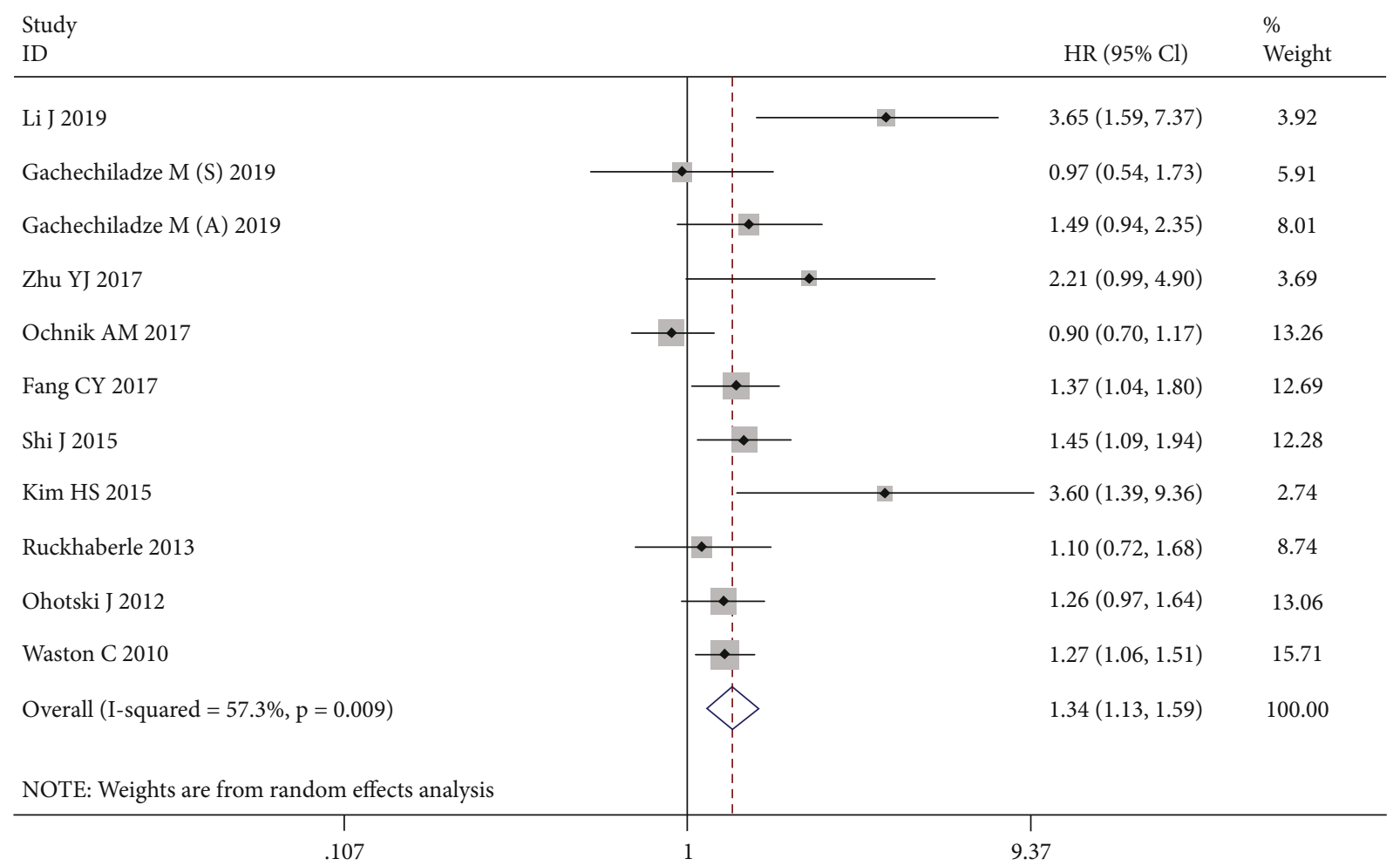

FIGURE 3: Forest plots of the overall outcomes for disease-free survival.

TABLE 3: Meta-analysis of SPHK1 and clinicopathological features in cancer patients.

\begin{tabular}{|c|c|c|c|c|c|c|}
\hline Categories & Cohorts (patients) & OR $(95 \% \mathrm{CI})$ & $I^{2}(\%)$ & $P_{h}$ & $Z$ & $P$ \\
\hline Age (young vs. old) & $21(4607)$ & $1.47(0.80-2.70)$ & 94.9 & $<0.001$ & 1.23 & 0.217 \\
\hline Gender (male vs. female) & $20(3515)$ & $1.02(0.87-1.19)^{\mathrm{F}}$ & 15.9 & 0.256 & 0.24 & 0.813 \\
\hline Clinical stage (I-II vs. III-IV) & $17(3951)$ & $2.07(1.39-3.09)$ & 79.6 & $<0.001$ & 3.56 & $<0.001$ \\
\hline Tumor invasion (T1-T2 vs. T3-T4) & $10(2062)$ & $2.16(1.47-3.18)$ & 52.8 & 0.025 & 3.91 & $<0.001$ \\
\hline Lymph node metastasis (negative vs. positive) & $17(2922)$ & $2.04(1.71-2.44)^{\mathrm{F}}$ & 33.3 & 0.090 & 7.88 & $<0.001$ \\
\hline Distant metastasis (negative vs. positive) & $12(2261)$ & $3.16(2.44-4.09)^{\mathrm{F}}$ & 0.0 & 0.632 & 8.72 & $<0.001$ \\
\hline Tumor size (small vs. large) & $14(2655)$ & $1.36(0.85-2.18)$ & 84.8 & $<0.001$ & 1.28 & 0.199 \\
\hline
\end{tabular}

All pooled ORs were calculated from random-effect model except for cells marked with (fixed ${ }^{\mathrm{F}}$ ). $P_{h}$ denotes $P$ value for heterogeneity based on $Q$ test; $P$ denotes $P$ value for statistical significance based on $Z$ test. OR: odds ratio; CI: confidence interval.

reported that SPHK1 promoted metastasis of cancer cells by inducing EMT, which was mediated by the FAK/AKT/ MMPs axis [19]. Furthermore, it has been suggested that S1P released by SPHK1 activity promotes EMT in cancer by inhibiting the Snail-matrix metalloproteinases and remodeling the glycocalyx signaling pathway [62].

Thus, this study demonstrated that high immunohistochemical expression of SPHK1 in cancer tissues may be associated with poor survival in human solid tumor patients, which will promote the development of a novel biomarker for the diagnosis and prognosis and targeted therapy of solid tumors. First of all, in addition to biopsy or surgical tissue, further research on whether this important marker is also detectable in other forms of patient samples, such as blood, will be very important for the early detection and diagnosis of tumors [15]. Second, SPHK1 expression can be used as a reliable biomarker to grade the prognosis of cancer patients [37]. Finally, targeting SPHK1 or its downstream targets with clinically available inhibitors would be effective for tumor therapy, such as increasing tumor sensitivity to chemotherapy $[36,63,64]$. Therefore, further preclinical and clinical development of SPHK1 inhibitors is necessary for the treatment of tumors [5].

Apart from the inspiring results, several limitations still should be noted in this quantitative meta-analysis. First, the population of the included studies was mainly concentrated in Asia, which affected the applicability of the results to some extent. Second, the inconsistent cut-off values for distinguishing between high and low expression of SPHK1 and the different analysis methods for evaluating the correlation between SPHK1 overexpression and prognosis may lead to different results of the included studies. Third, all of the included studies were retrospective cohort studies, in which positive results are more likely to be published than negative 


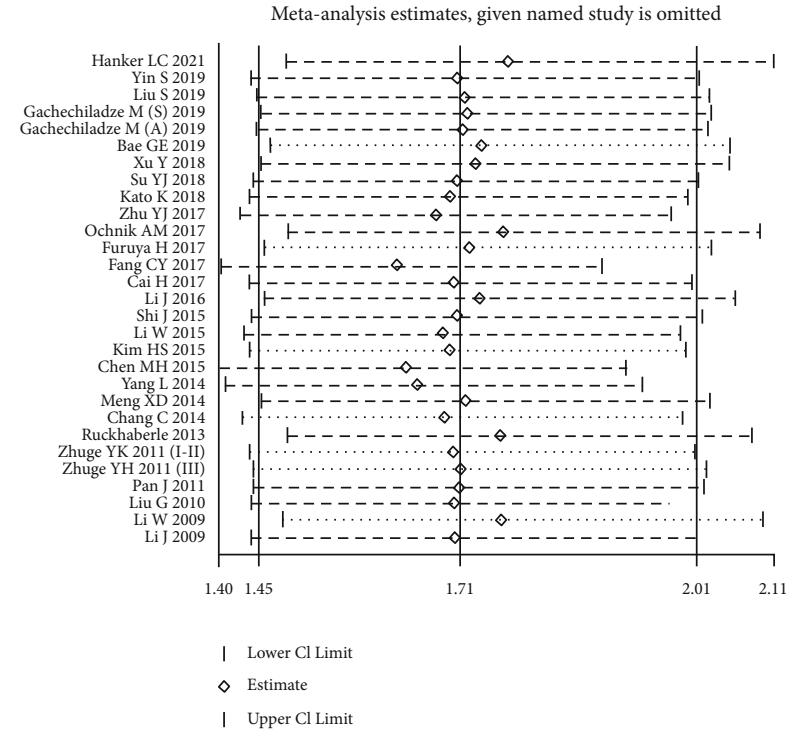

(a)

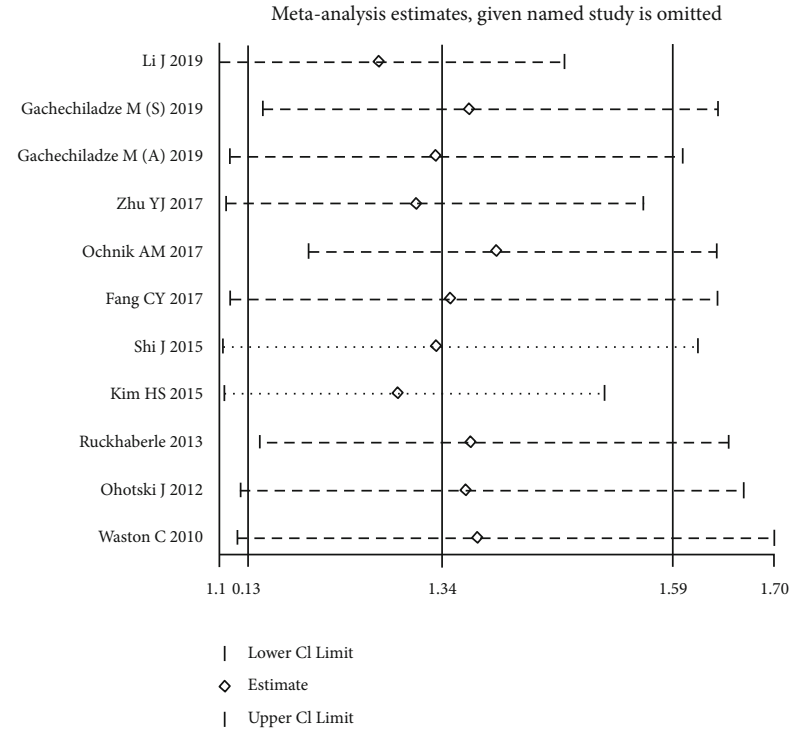

(b)

FIGURE 4: Effects of individual studies on pooled hazard ratios for SPHK1 expression and survival in solid tumors. (a) Result of sensitivity analysis for pooled overall survival estimation. (b) Result of sensitivity analysis for pooled disease-free survival estimation.

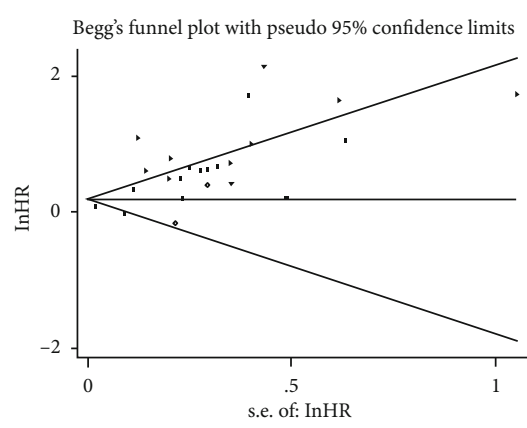

(a)

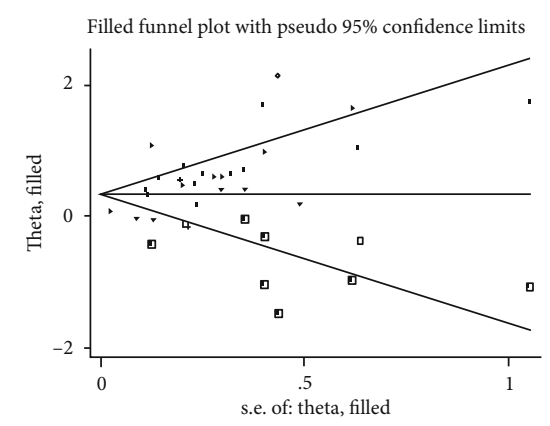

(b)

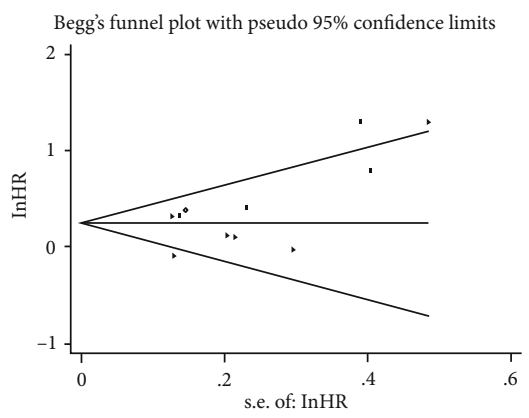

(c)

FIGURE 5: Begg's funnel plots for assessment of potential publication bias in studies of SPHK1 in patients with solid tumor. Each study is represented by one circle. The horizontal line represented the pooled effect estimate. (a) Funnel plot of publication bias for studies reporting overall survival. (b) Funnel plot adjusted with trim and fill methods for studies reporting overall survival. (c) Funnel plot of publication bias for studies reporting disease-free survival.

results. Fourth, as some studies did not directly provide the HRs, we had to estimate the HRs and 95\% CIs from the survival curves, which may cause some errors. Fifth, the publication bias of this study is a concern because articles with positive results are more likely to be published, which may exaggerate the connection between SPHK1 expression and adverse outcomes. Sixth, the quality of the evidence for both OS and DFS was low, which affected our confidence in the estimation of the effect. Finally, this meta-analysis was not prospectively registered in international prospective register of systematic reviews (PROSPERO) that can help reduce selective reporting of outcomes [65]. 
TABLE 4: Risk of bias assessment using the GRADE instrument.

\begin{tabular}{|c|c|c|c|c|c|c|c|c|}
\hline \multirow[b]{2}{*}{$\begin{array}{l}\begin{array}{l}\text { No. of } \\
\text { studies }\end{array} \\
\end{array}$} & \multirow[b]{2}{*}{ Study design } & \multicolumn{5}{|c|}{ Quality assessment } & \multirow[b]{2}{*}{ Quality } & \multirow[b]{2}{*}{ Importance } \\
\hline & & $\begin{array}{c}\text { Risk of } \\
\text { bias }\end{array}$ & Inconsistency & Indirectness & Imprecision & Other considerations & & \\
\hline \multicolumn{9}{|l|}{ OS } \\
\hline 29 & $\begin{array}{c}\text { Observational } \\
\text { studies }\end{array}$ & $\begin{array}{c}\text { Not } \\
\text { serious }\end{array}$ & Serious & Not serious & Not serious & $\begin{array}{l}\text { Publication bias strongly suspected; } \\
\text { strong association; all plausible } \\
\text { residual confounding would suggest } \\
\text { spurious effect, while no effect was } \\
\text { observed }\end{array}$ & $\underset{\text { Low }}{\bigoplus \bigoplus \bigcirc \bigcirc}$ & $\begin{array}{l}\text { Our } \\
\text { confidence } \\
\text { in the effect } \\
\text { estimate is } \\
\text { limited }\end{array}$ \\
\hline \multicolumn{9}{|l|}{ DFS } \\
\hline 11 & $\begin{array}{c}\text { Observational } \\
\text { studies }\end{array}$ & Serious & Serious & Not serious & Not serious & $\begin{array}{c}\text { Strong association; all plausible } \\
\text { residual confounding would suggest } \\
\text { spurious effect, while no effect was } \\
\text { observed }\end{array}$ & $\underset{\text { Low }}{\bigoplus \bigoplus \bigcirc \bigcirc}$ & $\begin{array}{l}\text { Our } \\
\text { confidence } \\
\text { in the effect } \\
\text { estimate is } \\
\text { limited }\end{array}$ \\
\hline
\end{tabular}

Question: "Is Upregulation of SPHK1 associated with poor prognosis in human solid tumors?".

\section{Conclusion}

In summary, high SPHK1 expression was associated with poor prognosis and served as a useful prognostic biomarker, which might be a promising therapeutic target for solid tumors. However, conclusions need to be treated with caution because of lack of high quality of evidence.

\section{Data Availability}

The data supporting this meta-analysis are from previously reported studies and datasets, which have been cited. The processed data are available within the article.

\section{Conflicts of Interest}

The authors declare that they have no conflict of interest.

\section{Acknowledgments}

This study was funded by Taizhou People's Hospital Medical Innovation Team Foundation (CXTDA201901) and Taizhou People's Hospital Mandatory Project (ZL202020 and ZL202029).

\section{References}

[1] F. Bray, J. Ferlay, I. Soerjomataram, R. L. Siegel, L. A. Torre, and A. Jemal, "Global cancer statistics 2018: GLOBOCAN estimates of incidence and mortality worldwide for 36 cancers in 185 countries," CA: a Cancer Journal for Clinicians, vol. 68, no. 6, pp. 394-424, 2018.

[2] R. L. Siegel, A. Jemal, R. C. Wender, T. Gansler, J. Ma, and O. W. Brawley, "An assessment of progress in cancer control," CA: a Cancer Journal for Clinicians, vol. 68, no. 5, pp. 329-339, 2018.

[3] R. L. Siegel, K. D. Miller, and A. Jemal, "Cancer statistics, 2018," CA: a Cancer Journal for Clinicians, vol. 68, no. 1, pp. 7-30, 2018.

[4] D. Feng, Q. Cao, D. Zhang et al., "Transcription factor E2F1 positively regulates interferon regulatory factor 5 expression in non-small cell lung cancer," Oncotargets and Therapy., vol. Volume 12, pp. 6907-6915, 2019.

[5] H. S. Kim, G. Yoon, J. Y. Ryu et al., "Sphingosine kinase 1 is a reliable prognostic factor and a novel therapeutic target for uterine cervical cancer," Oncotarget, vol. 6, no. 29, pp. 26746-26756, 2015.

[6] C. R. Gault and L. M. Obeid, "Still benched on its way to the bedside: sphingosine kinase 1 as an emerging target in cancer chemotherapy," Critical Reviews in Biochemistry and Molecular Biology., vol. 46, no. 4, pp. 342-351, 2011.

[7] S. Spiegel and S. Milstien, "Functions of the Multifaceted Family of Sphingosine Kinases and Some Close Relatives," Journal of Biological Chemistry., vol. 282, no. 4, pp. 2125-2129, 2007.

[8] B. Ogretmen, "Sphingolipid metabolism in cancer signalling and therapy," Nature Reviews Cancer., vol. 18, no. 1, pp. 3350, 2018.

[9] S. Spiegel and S. Milstien, "Sphingosine-1-phosphate: an enigmatic signalling lipid," Nature Reviews. Molecular Cell Biology, vol. 4, no. 5, pp. 397-407, 2003.

[10] J. A. Green, K. Suzuki, B. Cho et al., "The sphingosine 1phosphate receptor $\mathrm{S}_{2} \mathrm{P}_{2}$ maintains the homeostasis of germinal center B cells and promotes niche confinement," Nature Immunology, vol. 12, no. 7, pp. 672-680, 2011.

[11] A. Olivera, T. Kohama, L. Edsall et al., "Sphingosine kinase expression increases intracellular sphingosine-1-phosphate and promotes cell growth and Survival," The Journal of Cell Biology, vol. 147, no. 3, pp. 545-558, 1999.

[12] S. E. Alvarez, S. Milstien, and S. Spiegel, "Autocrine and paracrine roles of sphingosine-1-phosphate," Trends in Endocrinology \& Metabolism., vol. 18, no. 8, pp. 300-307, 2007.

[13] K. R. Johnson, K. Y. Johnson, H. G. Crellin et al., "Immunohistochemical distribution of sphingosine kinase 1 in normal and tumor lung Tissue," Journal of Histochemistry \& Cytochemistry., vol. 53, no. 9, pp. 1159-1166, 2005.

[14] M. M. Facchinetti, N. A. Gandini, M. E. Fermento et al., "The expression of sphingosine kinase-1 in head and neck Carcinoma," Cells, Tissues, Organs, vol. 192, no. 5, pp. 314-324, 2010.

[15] W. Li, C. Yu, J. Xia et al., "Sphingosine kinase 1 is associated with gastric cancer progression and poor survival of Patients," Clinical Cancer Research, vol. 15, no. 4, pp. 1393-1399, 2009. 
[16] M. Gachechiladze, T. Tichý, V. Kolek et al., "Sphingosine kinase-1 predicts overall survival outcomes in non-small cell lung cancer patients treated with carboplatin and navelbine," Oncology Letters, vol. 18, no. 2, pp. 1259-1266, 2019.

[17] J. Li, B. Zhang, Y. Bai, Y. Liu, B. Zhang, and J. Jin, "Upregulation of sphingosine kinase 1 is associated with recurrence and poor prognosis in papillary thyroid carcinoma," Oncology Letters, vol. 18, no. 5, pp. 5374-5382, 2019.

[18] S. Yin, Z. Miao, Y. Tan et al., "SPHK1-induced autophagy in peritoneal mesothelial cell enhances gastric cancer peritoneal dissemination," Cancer Medicine, vol. 8, no. 4, pp. 17311743, 2019.

[19] S. Liu, C. Xu, W. Wu et al., "Sphingosine kinase 1 promotes the metastasis of colorectal cancer by inducing the epithelialmesenchymal transition mediated by the FAK/AKT/MMPs axis," International journal of oncology., vol. 54, no. 1, pp. 41-52, 2019.

[20] Y. J. Su, J. X. Zhang, S. M. Li, X. H. Tan, and J. A. Huang, "Relationship of vasculogenic mimicry, SphK1 expression, and Cx43 expression to metastasis and prognosis in colorectal cancer," International Journal of Clinical and Experimental Pathology, vol. 11, no. 11, pp. 5290-5299, 2018.

[21] Y. Xu, B. Dong, J. Wang, J. Zhang, W. Xue, and Y. Huang, "Sphingosine kinase 1 overexpression contributes to sunitinib resistance in clear cell renal cell carcinoma," Oncoimmunology., vol. 7, no. 12, article e1502130, 2018.

[22] H. Cai, X. Xie, L. Ji, X. Ruan, and Z. Zheng, "Sphingosine kinase 1: a novel independent prognosis biomarker in hepatocellular carcinoma," Oncology Letters, vol. 13, no. 4, pp. 23162322, 2017.

[23] C. Y. Fang, C. W. Zhu, L. H. Yu, and Y. Y. He, "Expression of sphingosine kinase 1 in primary hepatocellular carcinoma tissues and its clinical significance[J]," Journal of Shenyang Medical College., vol. 19, no. 6, pp. 473-476, 2017.

[24] Y. J. Zhu, H. You, J. X. Tan et al., “Overexpression of sphingosine kinase 1 is predictive of poor prognosis in human breast cancer," Oncology Letters, vol. 14, no. 1, pp. 63-72, 2017.

[25] J. Li, H. Wu, W. Li et al., "Downregulated miR-506 expression facilitates pancreatic cancer progression and chemoresistance via SPHK1/Akt/NF- $\kappa \mathrm{B}$ signaling," Oncogene, vol. 35 , no. 42 , pp. 5501-5514, 2016.

[26] M. H. Chen, C. C. Yen, C. T. Cheng et al., "Identification of SPHK1 as a therapeutic target and marker of poor prognosis in cholangiocarcinoma," Oncotarget, vol. 6, no. 27, pp. 23594-23608, 2015.

[27] W. Li, Z. Tian, H. Qin et al., "High expression of sphingosine kinase 1 is associated with poor prognosis in nasopharyngeal carcinoma," Biochemical and Biophysical Research Communications, vol. 460, no. 2, pp. 341-347, 2015.

[28] J. Shi, Y. He, J. Sun et al., "The impact of sphingosine kinase 1 on the prognosis of hepatocellular carcinoma patients with portal vein tumor thrombus," Annals of Hepatology, vol. 14, no. 2, pp. 198-206, 2015.

[29] C. Chang, M. Xu, and J. Wang, "Expression level of sphingosine kinase 1 and nuclear factor- $\kappa \mathrm{B}$ p65 in non-small cell lung cancer and their relationship with tumor prognosis[J]," Tianjin Medical Journal., vol. 42, no. 4, pp. 305-308, 2014.

[30] Y. A. N. G. Lan, H. U. Honglin, D. E. N. G. Ying, and B. A. I. Yifeng, "Role of SPHK1 regulates multi-drug resistance of small cell lung cancer and its clinical signiifcance[J]," Chinese Journal of Lung Cancer., vol. 17, no. 11, pp. 769-777, 2014.
[31] J. Pan, Y. Tao, Z. Zhou et al., "An novel role of sphingosine kinase-1 (SPHK1) in the invasion and metastasis of esophageal carcinoma," Journal of Translational Medicine, vol. 9, no. 1, p. 157, 2011.

[32] Y. H. Zhuge, H. Q. Wang, and Y. Y. Wang, "Relationship between sphingosine kinase 1 expression and tumor invasion冈metastasis and prognosis in gastric cancer[J]," Natl Med J China., vol. 91, no. 39, pp. 2765-2768, 2011.

[33] G. Liu, H. Zheng, Z. Zhang et al., "Overexpression of sphingosine kinase 1 is associated with salivary gland carcinoma progression and might be a novel predictive marker for adjuvant therapy," BMC Cancer, vol. 10, no. 1, p. 495, 2010.

[34] C. Watson, J. S. Long, C. Orange et al., "High Expression of Sphingosine 1-Phosphate Receptors, $\mathrm{S}_{1} \mathrm{P}_{1}$ and $\mathrm{S}_{1} \mathrm{P}_{3}$, Sphingosine Kinase 1, and Extracellular Signal-Regulated Kinase-1/2 Is Associated with Development of Tamoxifen Resistance in Estrogen Receptor-Positive Breast Cancer Patients," The American Journal of Pathology, vol. 177, no. 5, pp. 22052215, 2010.

[35] J. Li, H. Guan, L. Gong et al., "Clinical significance of sphingosine Kinase-1 Expression in human astrocytomas progression and overall patient Survival," Clinical Cancer Research, vol. 14, no. 21, pp. 6996-7003, 2008.

[36] L. C. Hanker, A. el-Balat, Z. Drosos et al., "Sphingosinekinase-1 expression is associated with improved overall survival in high-grade serous ovarian cancer," Journal of Cancer Research and Clinical Oncology, vol. 147, no. 5, pp. 14211430, 2021.

[37] G. E. BAE, S. I. DO, K. KIM, J. H. PARK, S. CHO, and H. S. KIM, "Increased sphingosine kinase 1 expression predicts distant metastasis and poor outcome in patients with colorectal Cancer," Anticancer Research, vol. 39, no. 2, pp. 663-670, 2019.

[38] K. Kato, M. Shimasaki, T. Kato, N. Segami, and Y. Ueda, "Expression of sphingosine kinase-1 is associated with invasiveness and poor prognosis of oral squamous cell Carcinoma," Anticancer Research, vol. 38, no. 3, pp. 1361-1368, 2018.

[39] H. Furuya, Y. Shimizu, P. M. Tamashiro et al., "Sphingosine kinase 1 expression enhances colon tumor growth," Journal of Translational Medicine, vol. 15, no. 1, p. 120, 2017.

[40] A. M. Ochnik and R. C. Baxter, "Insulin-like growth factor receptor and sphingosine kinase are prognostic and therapeutic targets in breast cancer," BMC Cancer, vol. 17, no. 1, p. 820, 2017.

[41] X. D. Meng, Z. S. Zhou, J. H. Qiu, W. H. Shen, Q. Wu, and J. Xiao, "Increased SPHK1 expression is associated with poor prognosis in bladder cancer," Tumour Biology, vol. 35, no. 3, pp. 2075-2080, 2014.

[42] E. Ruckhäberle, T. Karn, C. Denkert et al., "Predictive value of sphingosine kinase 1 expression in neoadjuvant treatment of breast cancer," Journal of Cancer Research and Clinical Oncology, vol. 139, no. 10, pp. 1681-1689, 2013.

[43] J. Ohotski, J. S. Long, C. Orange et al., "Expression of sphingosine 1-phosphate receptor 4 and sphingosine kinase 1 is associated with outcome in oestrogen receptor-negative breast cancer," British Journal of Cancer, vol. 106, no. 8, pp. 14531459, 2012.

[44] L. Jiao, J. Wei, J. Ye, and C. Zhang, "Prognostic Value of Peroxiredoxin-1 Expression in Patients with Solid Tumors: a Meta-Analysis of Cohort Study," Disease Markers., vol. 2021, pp. 1-10, 2021. 
[45] J. F. Tierney, L. A. Stewart, D. Ghersi, S. Burdett, and M. R. Sydes, "Practical methods for incorporating summary timeto-event data into meta-analysis," Trials, vol. 8, no. 1, p. 16, 2007.

[46] A. Stang, "Critical evaluation of the Newcastle-Ottawa scale for the assessment of the quality of nonrandomized studies in meta-analyses," European Journal of Epidemiology., vol. 25, no. 9, pp. 603-605, 2010.

[47] H. Balshem, M. Helfand, H. J. Schünemann et al., "GRADE guidelines: 3. Rating the quality of evidence," Journal of Clinical Epidemiology., vol. 64, no. 4, pp. 401-406, 2011.

[48] A. Iorio, F. A. Spencer, M. Falavigna et al., "Use of GRADE for assessment of evidence about prognosis: rating confidence in estimates of event rates in broad categories of patients," $B M J$, vol. 350, no. mar16 7, article h870, 2015.

[49] D. Atkins, D. Best, P. A. Briss et al., "Grading quality of evidence and strength of recommendations," BMJ, vol. 328, no. 7454, p. 1490, 2004.

[50] J. P. T. Higgins, S. G. Thompson, J. J. Deeks, and D. G. Altman, "Measuring inconsistency in meta-analyses," BMJ, vol. 327, no. 7414, pp. 557-560, 2003.

[51] M. Egger, G. D. Smith, M. Schneider, and C. Minder, "Bias in meta-analysis detected by a simple, graphical test," $B M J$, vol. 315, no. 7109, pp. 629-634, 1997.

[52] P. Xia, L. Wang, P. A. B. Moretti et al., "Sphingosine Kinase Interacts with TRAF2 and Dissects Tumor Necrosis Factor- $\alpha$ Signaling," The Journal of Biological Chemistry, vol. 277, no. 10, pp. 7996-8003, 2002.

[53] B. W. Wattenberg, S. M. Pitson, and D. M. Raben, "The sphingosine and diacylglycerol kinase superfamily of signaling kinases: localization as a key to signaling function," Journal of Lipid Research, vol. 47, no. 6, pp. 1128-1139, 2006.

[54] S. M. Pitson, P. Xia, T. M. Leclercq et al., "Phosphorylationdependent translocation of sphingosine kinase to the plasma membrane drives its oncogenic signalling," Journal of Experimental Medicine., vol. 201, no. 1, pp. 49-54, 2005.

[55] K. Song, L. Dai, X. Long, W. Wang, and W. di, "Follicle-stimulating hormone promotes the proliferation of epithelial ovarian cancer cells by activating sphingosine kinase," Scientific Reports, vol. 10, no. 1, p. 13834, 2020.

[56] S. Sarkar, M. Maceyka, N. C. Hait et al., "Sphingosine kinase 1 is required for migration, proliferation and survival of MCF-7 human breast cancer cells," FEBS Letters., vol. 579, no. 24, pp. 5313-5317, 2005.

[57] A. Datta, S. Y. Loo, B. Huang et al., "SPHK1 regulates proliferation and survival responses in triple-negative breast cancer," Oncotarget, vol. 5, no. 15, pp. 5920-5933, 2014

[58] S. Q. LIU, Y. J. SU, M. B. QIN, Y. B. MAO, J. A. HUANG, and G. D. TANG, "Sphingosine kinase 1 promotes tumor progression and confers malignancy phenotypes of colon cancer by regulating the focal adhesion kinase pathway and adhesion molecules," International Journal of Oncology, vol. 42, no. 2, pp. 617-626, 2013.

[59] W. Lu and Y. Kang, "Epithelial-Mesenchymal Plasticity in Cancer Progression and Metastasis," Developmental Cell., vol. 49, no. 3, pp. 361-374, 2019.

[60] N. Myong, "Loss of E-cadherin and acquisition of vimentin in epithelial-mesenchymal transition are noble indicators of uterine cervix cancer Progression," Korean Journal of Pathology., vol. 46, no. 4, pp. 341-348, 2012.
[61] J. Long, Y. Xie, J. Yin, W. Lu, and S. Fang, "SphK1 promotes tumor cell migration and invasion in colorectal cancer," Tumour Biology, vol. 37, no. 5, pp. 6831-6836, 2016.

[62] Y. ZENG, X. H. YAO, Z. P. YAN, J. X. LIU, and X. H. LIU, "Potential signaling pathway involved in sphingosine-1-phosphate-induced epithelial-mesenchymal transition in cancer," Oncology letters., vol. 12, no. 1, pp. 379-382, 2016.

[63] J. Guillermet-Guibert, L. Davenne, D. Pchejetski et al., “Targeting the sphingolipid metabolism to defeat pancreatic cancer cell resistance to the chemotherapeutic gemcitabine drug," Molecular Cancer Therapeutics., vol. 8, no. 4, pp. 809-820, 2009.

[64] S. Acharya, J. Yao, P. Li et al., "Sphingosine kinase 1 signaling promotes metastasis of triple-negative breast Cancer," Cancer Research., vol. 79, no. 16, pp. 4211-4226, 2019.

[65] S. Sideri, S. N. Papageorgiou, and T. Eliades, "Registration in the international prospective register of systematic reviews (PROSPERO) of systematic review protocols was associated with increased review quality," Journal of Clinical Epidemiology., vol. 100, pp. 103-110, 2018. 